\title{
Spectrum Efficient GFDM Based on Faster Than Nyquist Signaling
}

\author{
Mariana Baracat de Mello, Luciano Leonel Mendes, Tiago Cardoso Barbosa
}

\begin{abstract}
Future mobile communication system will provide high data rates for a miscellany of new applications. Spectrum efficiency is a key performance indicator that must be considerably improved for the beyond $5 \mathrm{G}$ networks. In this paper, a a new approach to increase the waveform spectrum efficiency is investigated. This novel schemes combines the faster-thanNyquist signaling with generalized frequency division multiplexing, resulting in a scheme that can hold the main benefits of this innovative waveform with the high spectral efficiency gain. The scheme proposed in this paper abandons the orthogonality principle for the time-frequency grid, reducing the spacing between the subcarriers. The reduced subcarrier spacing does not affect the minimum distance among the transmitted sequences, if the Mazo's limit is respected and the data can be recovered by a non-linear receiver without bit error penalties. In this paper, maximum likelihood receiver will be used for a proof of conception, showing that the bit error performance is equivalent to the one achieved by orthogonal systems. The system will be integrated with a forward error control scheme, showing that the proposed scheme can be fully integrated with modern error control codes, while improving the spectrum efficiency by $20 \%$.
\end{abstract} 6G.

Index Terms-spectrum efficiency, GFDM, FTN, beyond 5G,

\section{INTRODUCTION}

The main design objectives for fifth generation (5G) of mobile communications networks are: 1) improvement of data rate and system capacity with the enhancement Mobile Broadband (eMBB); 2) support for latency sensitive applications with Ultra Reliable Low Latency Communications (URLLC), and; 3) improvement of number of connected devices and reduction in energy consumption with the massive Machine Type Communications (mMTC) [1]. Recently, the Third Generation Partnership Project (3GPP) has standardized orthogonal frequency division multiplexing (OFDM) as the waveform for an eMBB scenario [2]. Release 16, which is focusing on URLLC and industry 4.0 applications will also rely on OFDM [3]. Although 5G New Radio (NR) can achieve high data rates, the spectrum efficiency of the adopted waveform was not improved significantly from previous wireless networks. The high spectrum efficiency and high data rates observed in $5 \mathrm{G}$ Networks come from the use of massive multiple-input multiple-output (MIMO) and wider bandwidth

M. B. de Mello, L. L. Mendes and T. C. Barbosa are with Instituto Nacional de Telecomunicações (Inatel), Santa Rita do Sapucaí, MG, 37540-000, Brazil (email: marianam@gee.inatel.br, luciano@inatel.br and tiago.cardoso@inatel.br).

This work was also supported in part by CNPq-Brasil (grant no. 05085/2018-2), RNP/5G-RANGE project (grant no. H2020/20172019/777137) and MCTI (grant no. 01250.075415/2018-04) under the CRR/RNP joint project on 5G-IoT networks.

Digital Object Identifier: $10.14209 /$ jcis.2020.35 at both $3.5 \mathrm{GHz}$ and mmWave bands. One important scenario, which is not being widely discussed, is the enhancement Remote Area Communications (eRAC). This scenario can provide connectivity in uncovered or underserved areas and it is likely to exploit UHF bands for long-range coverage. Large bandwidth and massive MIMO, that are the main key feature for the high throughput in $5 \mathrm{G}$ networks, cannot be used in this frequency band.

Several researches has pointed that $6 \mathrm{G}$ networks will demand a waveform with higher spectrum efficiency and more flexibility than conventional orthogonal approaches can deliver [4]. For future evolution of beyond $5 \mathrm{G}$ networks, a more flexible waveform that does not rely on the orthogonality principle would provide an extra degree of freedom for the physical layer (PHY) layer. Among the possible waveforms, generalized frequency division multiplexing (GFDM) [5] has received some attention because it covers OFDM and singlecarrier frequency domain equalization (SC-FDE) as corner cases, while achieving good performance in terms of out-ofband emissions (OOBE) and bit error rate (BER) without significant complexity increment [6]. In this scheme, a data block composed of $N$ complex Quadrature Amplitude Modulation (QAM) symbols is divided into $K$ subcarriers carrying $M$ subsymbols each. GFDM uses circular filtering per subcarrier, meaning that the waveform block is contained within $N$ samples. A single prototype filter is circularly shifted in time and frequency to provide all impulse responses necessary to carry the $N$ data symbols. Slight modifications in the definition of time-frequency grid enable GFDM to employ faster than Nyquist (FTN) signaling principles, improving system spectral efficiency without introducing performance loss in terms of BER.

FTN signaling, investigated by J. E. Mazo [7], allows pulses transmission beyond the Nyquist limit, without suffering BER performance loss due to the higher intersymbol interference (ISI). According to [7], the transmitted data symbols carried by the prototype pulse shape can be packed $25 \%$ tighter in time-domain without suffering a decrease in the minimum Euclidean distance among the transmitted sequences. This value is known as the Mazo limit [8]. Above this limit, the ISI becomes very high and the minimum Euclidean distance among the data symbol is compromised.

The main goal of this paper is to modify the GFDM structure in order to allow it to exploit the FTN signaling, significantly increasing its spectrum efficiency. Usually, FTN is combined with orthogonal waveforms. The approach presented in this papers differs from the state of the art by applying compression to a non-orthogonal waveform, adding flexibility, 
better use of cyclic prefix (CP), and improving the frequency localization of FTN signaling. These features are important for future wireless communication systems since new approaches for exploiting the spectrum and new communication strategies will become necessary to address future challenging requirements.

If the Mazo's limit is respected, non-linear receivers can be used to recover the data without BER performance loss. For proof-of-concept proposes, a maximum likelihood (ML) receiver will be used in this paper, but more practical receivers can be developed in the future by the research community. This paper also considers the integration of the a forward error control (FEC) based on Polar code, showing that the proposed scheme can be easily integrated with modern error control techniques. The polar code was recently presented by Arikan [9] [10] as an efficient channel code that can achieve the channel capacity for discrete and continuous memoryless channels. Polar code can have a systematic structure, low complexity and high flexibility coding and decoding algorithms. Due to its attractive properties, the Polar code was chosen to protect the information transmitted on the control channel in 5G networks [11]. For these reasons, this coding scheme was selected to be integrated with the FTN-GFDM waveform proposed for performance analysis purposes. Both uncoded and coded BER performance will be analysed in this paper.

The remainder of this paper is organized as follows. Section II introduces the FTN signaling principles, while Section III presents a background on FTN integration with orthogonal waveforms. Section IV presents the principles of the GFDM waveform and, in Section V, GFDM is modified to exploit FTN principles both in time and frequency domains, which is the main contribution of this paper. In Section VI, the BER performance for uncoded and Polar coded FTN-GFDM is evaluated by simulations and compared with BER performance of orthogonal systems. Finally, Section VII brings the main conclusions of this paper.

\section{FASTER THAN NYQUiST}

FTN signaling is a promising technique that can improve the spectral efficiency of future mobile networks through time pulse acceleration, i.e., replacing the symbol period $T$ by $T^{\prime}<$ $T$ [7], where $T$ is the symbol period. The complex QAM symbols $d_{m}$, with duration $T$, are carried by the unitary energy transmission pulse $g(t)$ every $T^{\prime}$ and then sent through the channel, leading to

$$
x(t)=\sqrt{\tau} \sum_{m=0}^{M-1} d_{m} g\left(t-m T^{\prime}\right),
$$

where $M$ is the total number of symbols transmitted and $T^{\prime}=\tau T, 0<\tau<1$, is the accelerated symbol period. The parameter $\tau$, defined as accelerator factor, is responsible for squeezing the signal in time domain. The normalization factor $\sqrt{\tau}$ is used to keep transmit power constant for fair comparison with orthogonal schemes.

Considering the additive white Gaussian noise (AWGN) channel, the received signal is given by

$$
y(t)=x(t)+w(t) .
$$

On the receiver side, the signal is processed by an matched filter (MF) to extract an estimate of the transmitted symbols. Due to the overlap of the pulses, ISI is inserted into the signal. The signal $r(t)$ at the output of the MF can be written as

$$
\begin{aligned}
& r(t)=y(t) * g^{*}(-t) \\
&=\left(\sum_{m=0}^{M-1} d_{m} g\left(t-m T^{\prime}\right)\right) * g^{*}(-t)+\bar{w}(t),
\end{aligned}
$$

where $g^{*}(-t)$ is the MF impulse response and $\bar{w}(t)$ is the noise at the MF output. The signal at the MF output can be represented using matrix notation by

$$
\mathbf{r}=\mathbf{G d}+\overline{\mathbf{w}}
$$

where $\overline{\mathbf{w}}$ is the filtered noise vector, with zero mean and covariance matrix $\sigma^{2} \mathbf{G}$. The elements of the $\mathbf{G}$ matrix, given by

$$
G_{m, m^{\prime}}=\int_{-\infty}^{\infty} g\left(t-m T^{\prime}\right) g^{*}\left(t-m^{\prime} T^{\prime}\right) d t
$$

represent the ISI between the $m$ th and $m^{\prime}$ th symbols. Therefore, ISI depends on the prototype filter and the acceleration factor $\tau$ [12].

The minimum Euclidean distance among data vectors is a fundamental parameter for the BER evaluation [7][13]. The square of Euclidean distance between two symbol vectors, $\mathbf{d}$ and $\mathbf{d}^{\prime}$, is given by [13][14]

$$
\begin{gathered}
\mu^{2}=\frac{1}{2 \tau E_{b}} \int_{m=-\infty}^{\infty}\left|\sum_{m=-\infty}^{\infty} x_{d}(t)-x_{d^{\prime}}(t)\right|^{2} d t= \\
\frac{1}{2 \tau E_{b}} \int_{m=-\infty}^{\infty}\left|\sum_{m=-\infty}^{\infty}\left(d_{m}-d_{m}^{\prime}\right) g\left(t-m T^{\prime}\right)\right|^{2} d t= \\
\int_{m=-\infty}^{\infty}\left|\sum_{m=-\infty}^{\infty} e_{m} g\left(t-m T^{\prime}\right)\right|^{2} d t= \\
\int_{m=-\infty}^{\infty}\left(\operatorname{Re}^{2}\left\{\sum_{m=-\infty}^{\infty} e_{m} g\left(t-m T^{\prime}\right)\right\}+\right. \\
\int_{m=-\infty}^{\infty}\left[\left(\sum_{m=-\infty}^{\infty} g\left(t-m \tau T^{\prime}\right) \operatorname{Re}\left\{e_{m}\right\}\right)^{2}+\right. \\
\left.\left.\int_{m=-\infty}^{\infty} \sum_{m=-\infty}^{\infty} e_{m} g\left(t-m T^{\prime}\right)\right\}\right) d t= \\
\sum_{m=-\infty}^{\infty} \sum_{n=-\infty}^{\infty} \operatorname{Im}\left\{e_{m}^{\infty} \operatorname{Re}\left\{e_{m}\right\} \operatorname{Re}\left\{e_{n}\right\} g\left(t-m T_{n}\right\} g\left(t-m T^{\prime}\right) g\left(t-n T^{\prime}\right)+\right. \\
\left.\left.\left.\sum_{m=-\infty}^{\infty} \sum_{n=-\infty}^{\infty}\left[\operatorname{Re}\left\{e_{m}\right\} \operatorname{Re}\left\{e_{n}\right\}+\operatorname{Im}\left\{e_{m}\right\} \operatorname{Im}\left\{e_{n}\right\}\right] G_{m, n}, \quad(6) \operatorname{Im}\left\{e_{m}\right\}\right)^{\prime}\right)\right] d t=
\end{gathered}
$$

where $\mu$ is the Euclidean distance between the sequences $\mathbf{d}$ and $\mathbf{d}^{\prime}, e_{m}=\left(d_{m}-d_{m}^{\prime}\right) / \sqrt{2 \tau E_{b}}$ is the normalized difference between sequences. From (6), the minimum Euclidean distance can be numerically estimated by

$$
\mu_{\min }=\min _{\mathbf{e} \neq 0} \sqrt{\mu^{2}} .
$$


Assuming a binary modulation with unitary average energy and orthonormal pulses, the square of the minimum Euclidean distance is equal to 2, regardless of the pulse shape [15]. Mazo showed that for values of $\tau$ greater than 0.802 , the minimum Euclidean distance remains 2 for binary signaling with sinc pulses, allowing a data rate increase of approximately $25 \%$ over conventional orthogonal systems [7][8]. The value of $\tau$ for which the minimum Euclidean distance does not fall below the value obtained for orthogonal signaling is known as the Mazo limit [7]. Years later, this concept extended to non-binary signaling [16], other transmission pulses [14], and multicarrier systems, where it became known as the two-dimensional Mazo limit [8].

The advantage of the FTN system is that, for a given $\tau$ greater than the Mazo's limit, the minimum Euclidean distance is not affected and, therefore, there is no performance loss in terms of BER if the ISI can be properly removed in the receiver side. Pulse compression increases the complexity of the receiver because transmitted symbols can no longer be detected individually but need to be detected as a sequence. Linear detectors, such as zero forcing (ZF) and minimum mean square error (MMSE), have poor performance because they are not able to mitigate ISI. To achieve optimal performance, it is necessary to employ nonlinear detectors, such as the maximum likelihood sequence estimation (MLSE) [17]. The MLSE detector finds a vector $\hat{\mathbf{d}}$ that minimizes the Euclidean norm below

$$
\arg \min _{\hat{\mathbf{d}} \in \xi^{M}}\|\mathbf{r}-\mathbf{G} \hat{\mathbf{d}}\|^{2},
$$

where $\|$.$\| is the Euclidean norm and \xi^{M}$ is the set with all possible $J^{M}$ sequences of data symbols, where $J$ represents the modulation cardinality. The MLSE detector searches among all sequences for the sequence with the shortest Euclidean distance from the received vector $\mathbf{r}$. Although the complexity of this scheme is prohibitive for practical applications when $J$ and $M$ are large, the MLSE can be used to show that FTN can achieve the same BER performance than conventional orthogonal schemes.

\section{Spectrally EfFicient Frequency Division MultipleXing}

In [8], FTN signaling has also been studied in the frequency domain. The main idea of the spectrally efficient frequency division multiplexing (SEFDM) modulation is to reduce the spacing between the subcarriers, below the orthogonality limit, to increase the spectral efficiency [16]. However, this increase comes at the cost of increasing the detection complexity and performance penalties, due to the intercarrier interference (ICI) inserted in the signal. The time domain SEFDM signal is given by

$$
x(t)=\sqrt{\frac{\alpha}{T}} \sum_{k=0}^{K-1} d_{k} \exp \left(\frac{j 2 \pi k \alpha t}{T}\right),
$$

where $K$ is the number of subcarriers, $T$ is the period of the SEFDM symbol, $d_{k}$ is the QAM symbol for the $k$ th subcarrier, and $\alpha$, restricted to $0<\alpha \leq 1$, represents the frequency compression factor. Thus, the separation between the subcarriers is given by

$$
\Delta f=\frac{\alpha}{T} .
$$

For $\alpha=1$, the SEFDM corresponds to OFDM.

Due to overlapping of the subcarriers, the total bandwidth occupied by the SEFDM signal is reduced by a factor of $\left(\frac{1}{\alpha}-1\right)$ compared to OFDM [18]. Assuming AWGN channel, the received signal is given by

$$
y(t)=x(t)+w(t)
$$

The received signal given by (11) is applied to a bank of MF, each one centralized for a given subcarrier. Assuming perfect synchronism, the signal sampled at the MF output is given by

$$
\mathbf{r}=\mathbf{G d}+\overline{\mathbf{w}},
$$

where $\overline{\mathbf{w}}$ is the noise vector at the MF bank output, and $\mathbf{G}=\mathbf{C}^{\mathrm{H}} \mathbf{C}$ is the correlation matrix representing the ICI of the SEFDM system, with $\mathbf{C}$ being a modulation matrix given by

$$
C_{n, k}=\frac{1}{\sqrt{Q}} \exp \left[\frac{j 2 \pi k \alpha n}{Q}\right],
$$

where $Q=\phi K$ is the number of samples in time domain, $\phi \geq 1$ is the oversampling factor and $n=0.1, \ldots, Q-1$. Due to loss of orthogonality between subcarriers, samples at the MF output will contain ICI. Therefore, low complexity linear receivers, such as ZF and MMSE, also have poor BER performance in this situation [19][20]. On the other hand, the MLSE detector can be used to extract symbols from the received sequence and achieve optimal performance. The MLSE detector searches all possible symbol combinations and decides on the combination with the shortest Euclidean distance from the vector $\mathbf{r}$, thus

$$
\arg \min _{\hat{\mathbf{d}} \in \xi^{K}}\|\mathbf{r}-\mathbf{G} \hat{\mathbf{d}}\|^{2},
$$

where $\xi^{K}$ is the set of all $J^{K}$ data symbol combinations for the SEFDM signal. As a result, complexity grows exponentially with the $K$ and $J$ increase, which means that the MLSE implementation is no feasible in practice, but it can be use as proof-of-concept.

\section{Generalized Frequency Division Multiplexing}

In GFDM, each block transmits $N=K M$ data symbols divided into $K$ subcarriers, each one carrying $M$ subsymbols [5] [21]. GFDM uses circular convolution between the data symbols and the transmit pulse of each subcarrier, so the waveform has $N$ samples to represent the $K M$ data symbols. The prototype filter for $N$ samples length is rotated $K$ samples for each successive subsymbol. Thus, each data symbol $d_{k, m}$ is carried by a pulse given by

$$
g_{k, m}[n]=g\left[\langle n-m K\rangle_{N}\right] \exp \left(j 2 \pi \frac{k}{K} n\right),
$$

where $g[n]$ is the prototype filter impulse response. In this case, $g_{k, m}[n]$ represents the prototype filter shifted to the $k$ th 
subcarrier and $m$ th subsymbol. All filters impulse responses can be organized into a transmission matrix $\mathbf{A}$, defined by

$\mathbf{A}=\left[\begin{array}{lllllll}\mathbf{g}_{0,0} & \mathbf{g}_{1,0} & \cdots & \mathbf{g}_{K-1,0} & \mathbf{g}_{0,1} & \cdots & \mathbf{g}_{K-1, M-1}\end{array}\right]$,

where $\left[\mathbf{g}_{k, m}\right]_{n}=g_{k, m}[n]$.

The transmit signal $\mathbf{x}$ can be written as

$$
\mathbf{x}=\mathbf{A d}
$$

where $\mathbf{d}=\left[\begin{array}{lllllll}d_{0,0} & d_{1,0} & \cdots & d_{K-1,0} & d_{0,1} & \cdots & d_{K-1, M-1}\end{array}\right]^{\mathrm{T}}$ is the vector of data symbols. A cyclic prefix (CP) is inserted at the beginning of each GFDM block, mitigating interblock interference caused by multipath channels. After removing the $\mathrm{CP}$, the signal at the receiver is given by

$$
\mathbf{y}=\mathbf{H x}+\mathbf{w}
$$

where $\mathbf{w}$ represents the AWGN vector with zero mean and variance $\sigma^{2}$ and $\mathbf{H}$ is the channel circulant matrix based on the channel impulse response, $\mathbf{h}$. The matrix $\mathbf{H}$ represents the circular convolution matrix between the vector $\mathbf{x}$ and the impulse response $\mathbf{h}$.

Assuming that the receiver can estimate the channel impulse response, an linear detector can be used to recover the data symbols. Three MF, ZF and MMSE receive matrices [5][21] are defined as

$$
\mathbf{B}=\left\{\begin{array}{l}
\mathbf{A}^{\mathrm{H}} \mathbf{H}^{-1} \text { for } \mathrm{MF} \\
\mathbf{A}^{-\mathbf{1}} \mathbf{H}^{-1} \text { for } \mathrm{ZF} \\
\left(\sigma^{2} \mathbf{I}+\mathbf{A}^{\mathrm{H}} \mathbf{H}^{\mathrm{H}} \mathbf{H A}\right)^{-1} \mathbf{A}^{\mathrm{H}} \mathbf{H}^{\mathrm{H}} \text { for MMSE }
\end{array}\right.
$$

where $[\cdot]^{H}$ is the Hermitian matrix, $\mathbf{R}_{w}=\frac{\sigma^{2}}{E} \mathbf{I}_{\mathrm{N}}$ is the noise covariance matrix weighted by the signal energy and $\mathbf{I}_{N}$ is the $N \times N$ identity matrix. The estimated symbol vector $\hat{\mathbf{d}}$ is defined by

$$
\hat{\mathbf{d}}=\mathbf{B y} .
$$

The MF demodulator is the simplest detector. It maximizes the signal-to-noise ratio (SNR), but does not remove the interference completely, which results in BER performance losses for high SNR [22]. The applicability of the ZF demodulator depends on the properties of $\mathbf{A}$, which must be well conditioned and invertable. The ZF eliminates the selfinterference but can cause noise enhancement, affecting the BER performance at low SNR values. The MMSE demodulator mitigates the problem of low SNR noise enhancement and minimizes the impact of ISI and ICI on the high SNR and, therefore, MMSE can be considered a compromise between $\mathrm{MF}$ and $\mathrm{ZF}$.

When MF is employed, $\mathbf{G}=\mathbf{A}^{\mathrm{H}} \mathbf{A}$ can be used to evaluate the residual ISI and ICI on the received GFDM signal. The elements of $\mathbf{G}$ represent the correlation between $\mathbf{g}_{k, m}$ and $\mathbf{g}_{k^{\prime}, m^{\prime}}$, as shown in Fig. 1. The main diagonal of $\mathbf{G}$ represents the gains resulting from the modulation and demodulation processes at the desired data symbols position, and the other values represent interference. The ISI and ICI depend on the chosen prototype filter, hence, it is necessary to consider the compromise relationship between ISI and ICI when selecting it.

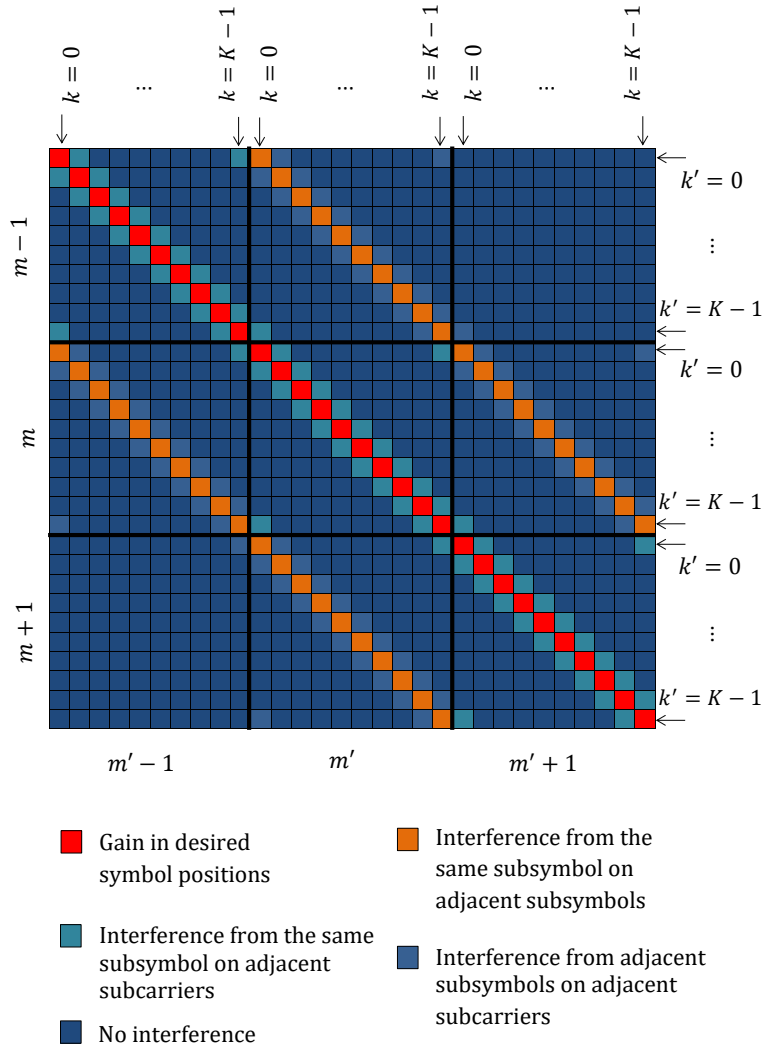

Fig. 1: Example of interference matrix for generic values of $M$ and $K$

Fig. 2 shows the simplified block diagram of a GFDM system [5]. The input data symbols are modulated by $\mathbf{A}$ in the GFDM modulator block. The samples at the output of this block represent the transmitted GFDM signal, given by (17). Then, the CP is added and the signal is applied to the channel. In the channel, the signal suffers distortions caused by multipaths and AWGN noise, as shown in (18). After the channel, the CP is removed and the signal goes through the GFDM demodulator, which can use three distinct approaches: MF, ZF, and MMSE, all shown in (19). At the output of the demodulator, symbols can be detected or delivered to the channel decoder [11].

\section{FREQUENCY FTN-GFDM}

The principles of FTN signaling can be integrated into the GFDM scheme by reformulating GFDM [5]. In order to achieve this goal, (15) must be rewritten as

$$
g_{k, m}[n]=g\left[\langle n-m K\rangle_{N}\right] \exp \left(j 2 \pi \frac{k M}{N} n\right) .
$$

Whereas the prototype filter has $\bar{N}$ samples, divided into $\bar{P}$ periods with $\bar{S}$ samples each, and that the subsymbols are separated by $\bar{K}$ samples in time and the subcarriers are 


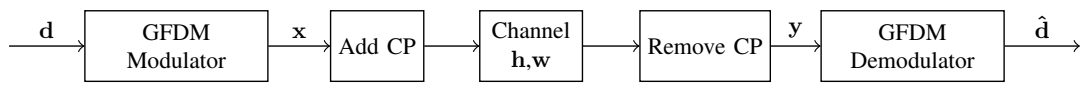

Fig. 2: GFDM simplified block diagram.

separated by $\bar{M}$ samples in frequency, the GFDM transmit vector can be written as

$x[n]=\sum_{k=0}^{K-1} \sum_{m=0}^{M-1} d_{k, m} g\left[\langle n-m \bar{K}\rangle_{\bar{N}}\right] \exp \left(j 2 \pi \frac{k \bar{M}}{\bar{N}} n\right)$,

with $n=0, \cdots, \bar{N}-1$. For the GFDM signal to be squeezed in the frequency domain, it is necessary to violate the Nyquist criterion, i.e., $\bar{K}=\bar{S}$ and $\bar{M}<\bar{P}$. The squeezing factor in frequency domain is defined as

$$
v_{f}=\frac{\bar{M}}{\bar{P}}
$$

Consequently, the new values of $K$ and $M$ are evaluated as

$$
K=\frac{\bar{P} \bar{S}}{\bar{M}}=\frac{\bar{S}}{v_{f}}=\left\lfloor\frac{\bar{N}}{\bar{M}}\right\rfloor,
$$

and

$$
M=\frac{\bar{P} \bar{S}}{\bar{K}}=\bar{P}
$$

Replacing the new values of $K$ and $M$ and $v_{f}$ in (22) leads to

$$
x[n]=\sqrt{v_{f}} \sum_{k=0}^{K-1} \sum_{m=0}^{M-1} d_{k, m} g\left[\langle n-m \bar{S}\rangle_{\bar{N}}\right] \exp \left(j 2 \pi \frac{k v_{f}}{\bar{S}} n\right),
$$

where the constant $\sqrt{v_{f}}$ keeps the transmission power constant and $n=0, \cdots, \bar{N}-1$.

Due to the high spectral efficiency of the FTN-GFDM signal, the density of data symbols per sample of the block, $\frac{N}{N}$, becomes greater than 1 . If $v_{f}=1$ the signal corresponds to the conventional GFDM, and the Nyquist criterion is respected.

It is important to note that if the value of the argument in (24) is not an integer, there will be a loss in the density of data symbols per sample of the block due to the floor operator. For the correct normalization of the signal's power, the value of the power normalization factor must be corrected, resulting in

$$
\bar{v}_{f}=\frac{\bar{S}}{K} .
$$

Finally, the FTN-GFDM signal is defined as

$$
\bar{x}[n]=\sqrt{\bar{v}_{f}} \sum_{k=0}^{K-1} \sum_{m=0}^{M-1} d_{k, m} g\left[\langle n-m \bar{S}\rangle_{\bar{N}}\right] \exp \left(j 2 \pi \frac{k v_{f}}{\bar{S}} n\right),
$$

with $n=0, \cdots, \bar{N}-1$. Fig. 3 shows the block diagram of the FTN-GFDM transmitter.

Spectral efficiency is an important parameter in evaluating the performance of a system. Considering the FTN-GFDM with $J$-QAM constellation, the data rate is given by

$$
R_{b}=\frac{R_{c} M K}{\left(\bar{P} \bar{S}+N_{\mathrm{CP}}\right) T_{s}},
$$

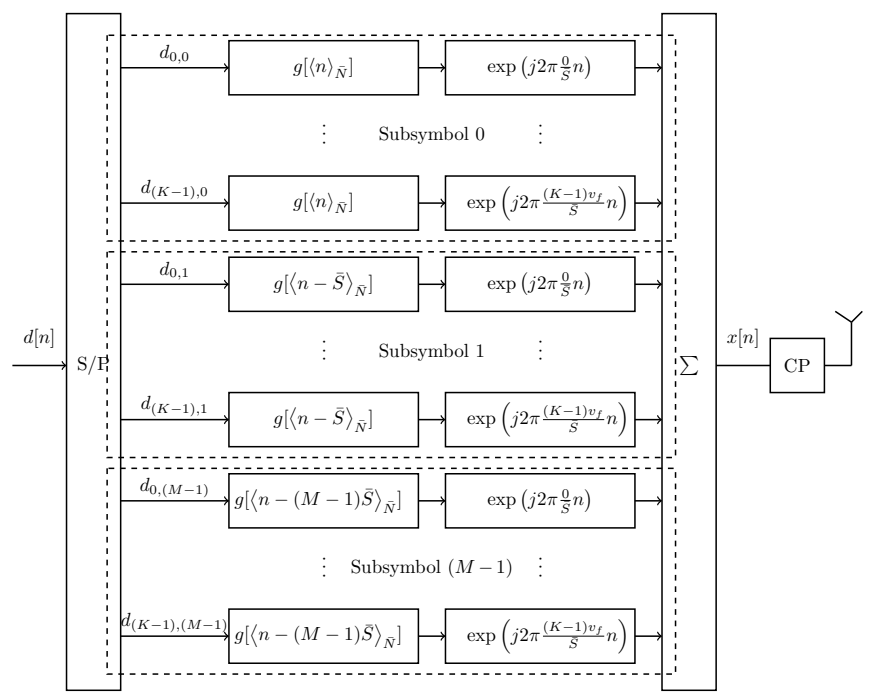

Fig. 3: FTN-GFDM transmission block diagram.

where $R_{c}$ is the FEC coding rate, $N_{\mathrm{CP}}$ is the number of samples used as CP and $T_{s}$ is the sampling period in seconds. The occupied bandwidth is given by

$$
B_{w}=\frac{1}{\log _{2}(J) T_{s}} .
$$

Thus, the spectral efficiency is defined as

$$
\eta=\frac{R_{c} M K \log _{2}(J)}{\bar{P} \bar{S}+N_{\mathrm{CP}}} .
$$

Considering the FTN-GFDM signal at (28), the received vector is given by

$$
\mathbf{y}=\mathbf{H} \overline{\mathbf{x}}+\mathbf{w} .
$$

Fig. 4 illustrates the block diagram of the FTN-GFDM communication chain. Thus, the FTN-GFDM modulator shown in Fig. 3 is integrated with channel, demodulator and detector blocks to complete the FTN-GFDM system.

The reception process consists of two blocks: demodulator, which also performs equalization, and detector. First, equalization, assuming that the receiver can estimate the channel impulse response, is combined with MF to decouple the subcarriers, leading to

$$
\mathbf{r}=\mathbf{A}^{\mathrm{H}} \mathbf{H}^{-1} \mathbf{H} \mathbf{A d}+\mathbf{A}^{\mathrm{H}} \mathbf{H}^{-1} \mathbf{w}=\mathbf{G d}+\overline{\mathbf{w}},
$$

where $\mathbf{G}=\mathbf{A}^{\mathrm{H}} \mathbf{A}$ is the interference matrix that represents ICI and ISI in the FTN-GFDM signal. The filtered noise vector $\overline{\mathbf{w}}=\mathbf{A}^{\mathrm{H}} \mathbf{H}^{-1} \mathbf{w}$ has correlated samples due to the nonorthogonality of the MF matrix. MF maximizes the SNR so that the detector can retrieve the transmitted symbols with an acceptable BER.

An important issue in the reception process is the conditioning of the $\mathbf{G}$ matrix, since the performance of the 


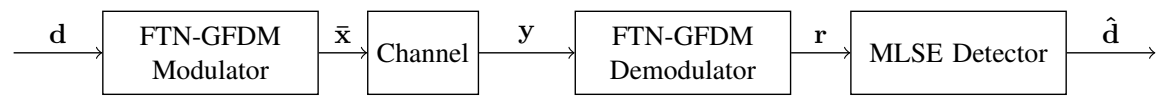

Fig. 4: Block diagram of the FTN-GFDM transceiver.

system is affected by ill conditioning. A matrix is said to be ill conditioned when its rows or columns are correlated and, consequently, its determinant is close to zero. Thus, the determinant of a matrix can be an indicator of the conditioning of a matrix.

Due to ISI and ICI present in the FTN-GFDM system, the G matrix is said to be ill correlated, since the magnitude of the elements that represent the cross-correlation between the subcarriers/subsymbols is different from zero. Thus, the conditioning of the FTN-GFDM system deteriorates with the reduction of the frequency squeezing factor, increment of the number of subcarriers and subsymbols in the system, or choice of the prototype filter used in the A matrix. Ill conditioning of a matrix leads to the sensitivity of the system to small disturbances and unstable matrix inversion, which affects the applicability of linear detectors such as ZF in FTN-GFDM systems.

Due to the $\mathbf{G}$ conditioning characteristics, the MLSE detector is used to recover the data symbols minimizing the BER. The computational complexity of MLSE in terms of complex multiplications is $O\left(N^{2} J^{N}\right)$. Therefore, the complexity increases significantly with $N$ and the QAM constellation size, hindering its use in practical cases. However, this receiver will be employed to evaluate the best BER performance that can be achieved by the proposed system. This result can be seen as a benchmark for sub-optimal receivers with lower complexity. Hence, the results achieved in the next section is a proof-ofconcept, showing that the FTN-GFDM can increase the data rate without introducing BER performance degradation.

\section{BER Performance Evaluation}

The uncoded and coded BER performance of the proposed FTN-GFDM system is evaluated by numerical simulations. Three channel models were considered: AWGN, frequencyselective channel (FSC) and time-variant channel (TVC) with a single Rayleigh tap that changes at every FTN-GFDM block. Table I presents the channel parameters, while Table III presents the FTN-GFDM parameters. Simulations assume that the channel is known on the receiver side and that the synchronization is perfect. Table II presents the software and hardware specifications of the device used in the simulations.

TABLE I: Channel models

\begin{tabular}{|c|c|}
\hline Channel & Impulse response \\
\hline AWGN & $\mathbf{h}_{\mathrm{AWGN}}=1$ \\
\hline Invariant FSC & $\mathbf{h}_{\mathrm{FSC}}=\left[\begin{array}{llll}1 & 0.4 & 0.2 & 0.08\end{array}\right]$ \\
\hline Time-variant channel & $\mathbf{h}_{\mathrm{TVC}}=h, h \sim \mathcal{C N}(0,1)$ \\
\hline
\end{tabular}

The simulated uncoded BER is compared with the theoretical curves. The analytical BER expressions considering
TABLE II: Software and hardware specifications

\begin{tabular}{lc}
\hline & Specification \\
\hline Operating system & Windows 10 Pro \\
CPU & Intel Core i5 $2.20 \mathrm{GHz}$ \\
Memory & 8 GB RAM \\
Simulation software & MATLAB R2016a \\
\hline
\end{tabular}

binary phase shift keying (BPSK) modulation under AWGN, frequency selective and time variant channels are, respectively, given by [5]

$$
\begin{gathered}
P_{\mathrm{AWGN}}(e)=\frac{1}{2} \operatorname{erfc}\left(\sqrt{\frac{E_{b}}{N_{0}}}\right), \\
P_{\mathrm{FSC}}(e)=\frac{1}{2 K} \sum_{k=0}^{K-1} \operatorname{erfc}\left(\sqrt{\frac{|\mathcal{H}[k]|^{2} E_{b}}{N_{0}}}\right), \\
P_{\mathrm{TVC}}(e)=\frac{1}{2}\left(1-\sqrt{\frac{\frac{E_{b}}{N_{0}}}{\frac{E_{b}}{N_{0}}+1}}\right),
\end{gathered}
$$

where $\mathcal{H}$ is the channel frequency response, $E_{b}$ is the average energy per bit and $N_{0}$ is the noise power density.

Two prototype filters are considered: $i$ ) Dirichlet; and $i i$ ) rect. Table III shows the FTN-GFDM parameters used in the simulations. It is important to note that for other configurations with higher $K$ and $M$ values, the MLSE complexity becomes prohibitive. Therefore, the computational simulations in this paper will be restricted to the values presented in Table III.

TABLE III: FTN-GFDM system for the simulations.

\begin{tabular}{lc}
\hline Parameter & Description \\
\hline Mapping & BPSK \\
Number of periods $(\bar{P})$ & 3 \\
Number of samples per period $(\bar{S})$ & 5 \\
Subcarrier distance factor $\left(v_{f}\right)$ & 0.8 \\
Number of subsymbols $(M)$ & 3 \\
Number of subcarriers $(K)$ & 6 \\
FEC & Polar coding \\
FEC codeword legth & $\frac{1}{2}$ \\
FEC coding rate & Successive Interference Cancellation \\
FEC decoder & 14 bits punctured \\
\hline
\end{tabular}

The chosen parameters lead to $\bar{S} / v_{f}=5 / 0.8=6.25$, which is not an integer value. Due to the rounding, there is a loss in 
data density. Therefore, the transmission signal normalization is based on $\bar{v}_{f}=5 / 6=0.833$. Consequently, the data symbol density per sample of the FTN-GFDM block is 1.2.

The spectral efficiency for the proposed FTN-GFDM system can be evaluated from (31), resulting in $\eta=1.2 \mathrm{bits} / \mathrm{s} / \mathrm{Hz}$ for the uncoded case and $\eta=0.6 \mathrm{bits} / \mathrm{s} / \mathrm{Hz}$ for the coded case. This means that it is possible to increase the spectral efficiency by $20 \%$ compared with the Nyquist signaling, without increasing the modulation order, energy, or weakening the codeword.

A reference BER is established for each channel to measure the performance gain provided by the Polar code: $10^{-6}$ for AWGN, $10^{-5}$ for FSC e $3 \times 10^{-3}$ for TVC.

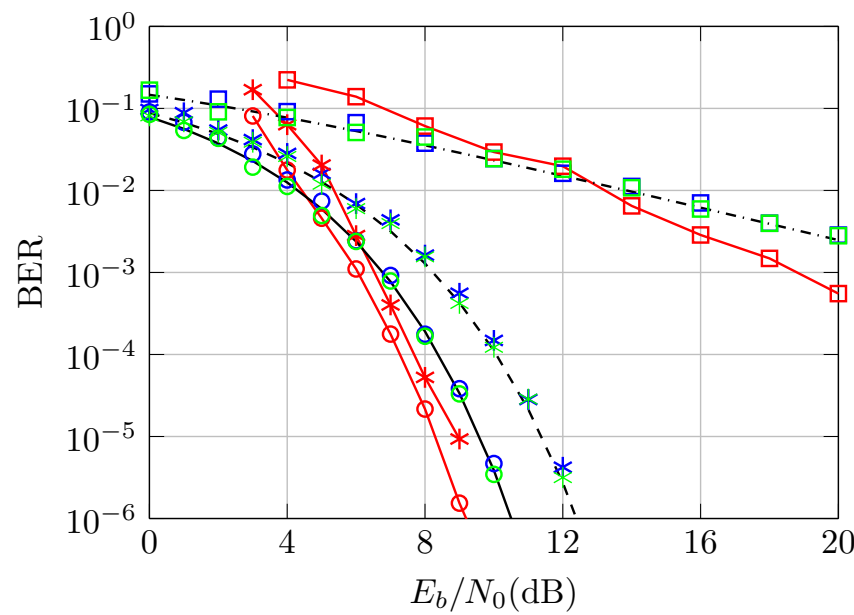

\begin{tabular}{|l|l|}
\hline o FTN-GFDM AWGN & - FTN-GFDM AWGN polar \\
$*$ FTN-GFDM FSC & $*$ FTN-GFDM FSC polar \\
aFTN-GFDM TVC & - FTN-GFDM TVC polar \\
○ GFDM AWGN & - Theo. BPSK AWGN \\
$*$ GFDM FSC & -- Theo. BPSK FSC \\
$\square$ GFDM TVC & --- Theo. BPSK TVC
\end{tabular}

Fig. 5: BER for FTN-GFDM BPSK with Dirichlet pulse. Assuming uncoded and coded cases with MLSE detection.

Fig. 5 considers the FTN-GFDM with Dirichlet prototype filter. System performance is evaluated in terms of BER for the coded and uncoded signal. The FTN-GFDM uncoded BER performance matches the theoretical curves, showing that the proposed system can improve the spectral efficiency without performance loss over all analyzed channels. As expected, Polar code improved the BER performance, showing that the MLSE can be seamlessly integrated with the Polar code. Considering the reference BER values, performance gains are approximately $1.5 \mathrm{~dB}$ for the AWGN channel, $2.5 \mathrm{~dB}$ for FSC, and $2.3 \mathrm{~dB}$ for TVC.

Fig. 6 shows the coded and uncoded BER performance considering the rect prototype filter. Once again, the simulation results for uncoded BER match the theoretical curves, showing that FTN-GFDM can increase the data rate and improve the spectrum efficiency without any BER penalty for different pulse-shapes. Also, the polar code provides a considerable improvement in the BER performance. The gain was approximately $3.5 \mathrm{~dB}$ for the AWGN channel, $4 \mathrm{~dB}$ for the FSC

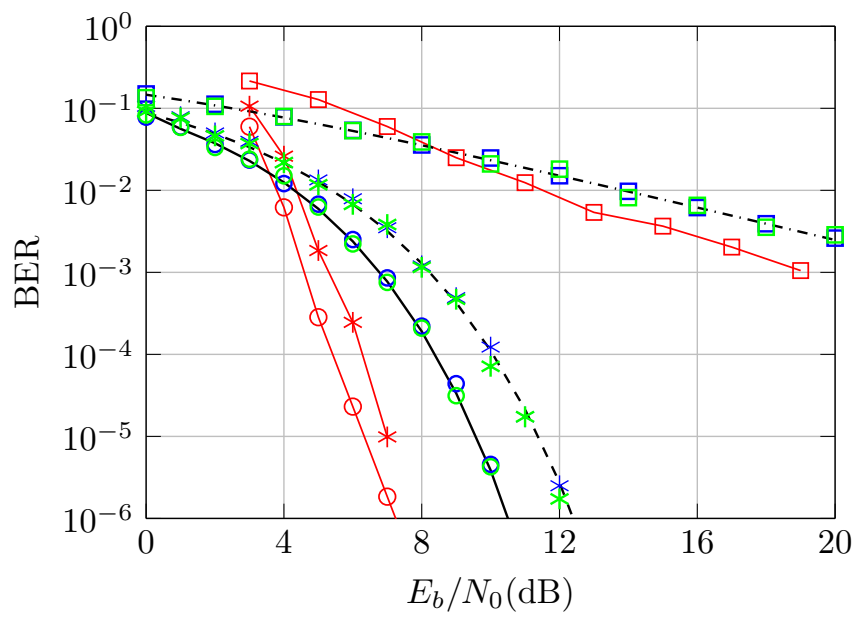

\begin{tabular}{|c|c|}
\hline o FTN-GFDM AWC & - - FTN-GFDM AWGN Pola \\
\hline * FTN-GFDM FSC & $*$ FTN-GFDM FSC Polar \\
\hline$\square$ FTN-GFDM TVC & $\square$ FTN-GFDM TVC Polar \\
\hline ○ GFDM AWGN & — Theo. BPSK AWGN \\
\hline$*$ GFDM FSC & - - - Theo. BPSK FSC \\
\hline$\square$ GFDM TVC & -.. Theo. BPSK TVC \\
\hline
\end{tabular}

Fig. 6: BER for FTN-GFDM BPSK with rect pulse. Assuming uncoded and coded cases with MLSE detection.

and $3.5 \mathrm{~dB}$ for the TVC. This shows that the Polar code performance also depends on the correlations introduced by the prototype pulse. The rect filter causes less ICI to the system, since the amount of energy that flows to neighboring symbols, evaluated by

$$
E_{\text {int }}=\sum_{m=0}^{N-1} \sum_{\substack{n=0 \\ n \neq m}}^{N-1} \frac{\left|G_{m, n}\right|}{\bar{v}_{f} N},
$$

is smaller with the rect filter. To ensure that the comparison is made with equivalent levels of interference, the energy is normalized so that the sum of the main diagonal of $\mathbf{G}$ is equal to 1 . Thus, the energy spent with ISI and ICI is $E_{\text {int }}=0.9843$ for rect prototype filter and $E_{\text {int }}=1.5374$ for Dirichlet, explaining the difference in coded BER performance.

Figs. 5 and 6 also bring the BER performance for conventional GFDM using the same parameters employed for the FTN-GFDM, showing that the later does not introduces performance loss when compared with the former. Therefore, the simulated GFDM system has $M=3$ and $K=5$, and the prototype filters are Dirichlet and rect. These parameters were obtained by making $v_{f}=1$, leading to $M=P$.

Thus, the proposed FTN-GFDM scheme can be used in scenarios where high robustness and efficiency are required and high number of transmit and receive antennas cannot be employed. The MLSE complexity is prohibitive in most practical situations, but it shows that the system proposed in this paper achieves higher spectrum efficiency without introducing BER performance losses. 


\section{CONCLUSION}

The FTN-GFDM system proposed in this paper combines the main advantages of FTN with GFDM. This solution is interesting in scenarios where high spectrum efficiency are necessary, but the number of transmit and receive antennas cannot be high, such as eRAC operating in VHF and UHF bands in remote areas. The FTN-GFDM BER performance matches the performance of orthogonal signaling. It has also been shown that Polar code can be seamlessly integrated with the FTN-GFDM system, further improving the overall performance. The MLSE detector used in this paper has very high complexity, but it shows that that the proposed scheme does not introduces BER performance losses. The MLSE performance also can be used as a benchmark for sub-optimal detector, with lower complexity and more practical appeal.

\section{REFERENCES}

[1] P. Pirinen, "A brief overview of 5G research activities," in 1st Int. Conf. 5G Ubiquitous Connectivity, Nov. 2014, pp. 17-22, doi:10.4108/icst.5gu.2014.258061.

[2] 3GPP, "5G; NR; Physical Layer General Description," 3rd Generation Partnership Project (3GPP), Tech. Specification (TS) 38.201, 2018, version 15.0.0.

[3] 3GPP, "Study on physical layer enhancements for NR ultra-reliable and low latency case (URLLC)," 3rd Generation Partnership Project (3GPP), Tech. Rep. (TR) 38.824, 2019, version 16.0.0.

[4] M. Z. Chowdhury, M. Shahjalal, S. Ahmed, and Y. M. Jang, "6G wireless communication systems: Applications, requirements, technologies, challenges, and research directions," IEEE Open J. Commun. Soc., vol. 1, pp. 957-975, 2020, doi:10.1109/OJCOMS.2020.3010270.

[5] L. Mendes, N. Michailow, M. Matthé, I. Gaspar, D. Zhang, and G. Fettweis, GFDM: Providing Flexibility for the 5G Physical Layer: A Research and Development Perspective. Springer, May 2016.

[6] D. Zhang, M. Matthé, L. L. Mendes, and G. Fettweis, "A study on the link level performance of advanced multicarrier waveforms under MIMO wireless communication channels," IEEE Trans. Wireless Commun., vol. 16, no. 4, pp. 2350-2365, Apr. 2017, doi:10.1109/TWC.2017.2664820.

[7] J. E. Mazo, "Faster-than-Nyquist signaling," Bell Syst. Tech. J, vol. 54, no. 8, pp. 1451-1462, Oct. 1975 , doi:10.1002/j.15387305.1975.tb02043.x.

[8] F. Rusek and J. B. Anderson, "The two dimensional Mazo limit," in IEEE Int. Symp. Inf. Theory, Sept. 2005, pp. 970-974, doi:10.1109/ISIT.2005.1523482.

[9] E. Arikan, "Channel polarization: A method for constructing capacityachieving codes for symmetric binary-input memoryless channels," IEEE Trans. Inf. Theory, vol. 55, no. 7, pp. 3051-3073, July 2009, doi:10.1109/TIT.2009.2021379.

[10] E. Saşoğlu, E. Telatar, and E. Arikan, "Polarization for arbitrary discrete memoryless channels," in IEEE Inf. Theory Workshop, Oct. 2009, pp. 144-148, doi:10.1109/ITW.2009.5351487.

[11] S. A. Hashemi, M. Mondelli, S. Hassani, C. Condo, R. Urbanke, and W. J. Gross, "Decoder partitioning: Towards practical list decoding of polar codes," IEEE Trans. Commun., vol. 66, no. 9, pp. 3749-3759, May 2018, doi:10.1109/TCOMM.2018.2832207.

[12] E. Bedeer, M. H. Ahmed, and H. Yanikomeroglu, "A very low complexity successive symbol-by-symbol sequence estimator for fasterthan-Nyquist signaling," IEEE Access, vol. 5, pp. 7414-7422, 2017, doi:10.1109/ACCESS.2017.2663762.

[13] J. Fan, S. Guo, X. Zhou, Y. Ren, G. Y. Li, and X. Chen, "Faster-thanNyquist signaling: An overview," IEEE Access, vol. 5, pp. 1925-1940, 2017, doi:10.1109/ACCESS.2017.2657599.

[14] A. D. Liveris and C. N. Georghiades, "Exploiting faster-than-Nyquist signaling," IEEE Trans. Commun., vol. 51, no. 9, pp. 1502-1511, Sep. 2003, doi:10.1109/TCOMM.2003.816943.

[15] J. B. Anderson, F. Rusek, and V. Öwall, "Faster-than-Nyquist signaling," Proc. IEEE, vol. 101, no. 8, pp. 1817-1830, Aug. 2013, doi:10.1109/JPROC.2012.2233451
[16] T. Xu and I. Darwazeh, "M-QAM signal detection for a non-orthogonal system using an improved fixed sphere decoder," in 9th IEEE Int. Symp. Commun. Syst., Netw. and Digit. Signal Process. (CSNDSP), July 2014, pp. 623-627, doi:10.1109/CSNDSP.2014.6923903.

[17] G. Forney, "Maximum-likelihood sequence estimation of digital sequences in the presence of intersymbol interference," IEEE Trans. Inf. Theory, vol. 18, no. 3, pp. 363-378, May 1972, doi:10.1109/TIT.1972.1054829.

[18] I. Kanaras, A. Chorti, M. Rodrigues, and I. Darwazeh, "A new quasioptimal detection algorithm for a non orthogonal spectrally efficient FDM," in 9th Int. Symp. Commun. and Inf. Technol., Sept. 2009, pp. 460-465, doi:10.1109/ISCIT.2009.5341206.

[19] I. Darwazeh and M. Rodrigues, "A spectrally efficient frequency division multiplexing based communications system," in Proc. 8th Int. OFDMWorkshop, Hamburg, Germany, Sept. 2003, pp. 70-74.

[20] I. Kanaras, A. Chorti, M. R. D. Rodrigues, and I. Darwazeh, "A combined MMSE-ML detection for a spectrally efficient non orthogonal FDM signal," in 5th Int. Conf. Broadband Commun., Netw. and Syst., Sept. 2008, pp. 421-425, doi:10.1109/BROADNETS.2008.4769119.

[21] G. Fettweis, M. Krondorf, and S. Bittner, "GFDM - generalized frequency division multiplexing," in IEEE 69th Veh. Technol. Conf., Apr. 2009, pp. 1-4, doi:10.1109/VETECS.2009.5073571.

[22] A. Farhang, N. Marchetti, and L. E. Doyle, "Low complexity GFDM receiver design: A new approach," in IEEE Int. Conf. Commun. (ICC), June 2015, pp. 4775-4780, doi:10.1109/ICC.2015.7249078.

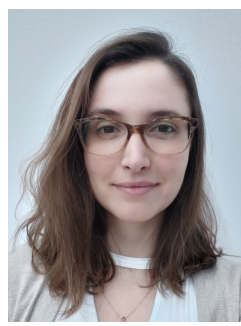

Mariana Baracat de Mello received the B.Sc. and M.Sc. degrees in Electrical Engineering from the National Institute of Telecommunications (Inatel), Brazil in 2015 and 2019, respectively. Her research interests include multicarrier modulation techniques for futures wireless systems, sphere decoder algorithms, and artificial intelligence schemes applied to communications.

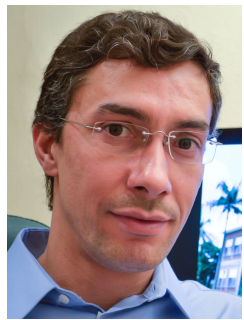

Luciano Leonel Mendes received the B.Sc. and M.Sc. degrees from Inatel, Brazil, in 2001 and 2003 , respectively, and the Doctor degree from Unicamp, Brazil, in 2007, all in electrical engineering. Since 2001, he has been a professor with Inatel. He has led several research projects funded by FAPEMIG, FINEP, and BNDES. From 2013 to 2015, he was a Visiting Researcher with the Technical University of Dresden in the Vodafone Chair Mobile Communications Systems. His main research interests include solutions for the physical layer of the future fifth generation of mobile communication systems. He has also been a CNPq level 2 Research Fellow since 2016.

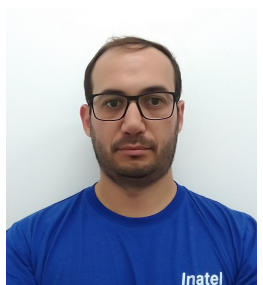

Tiago Barbosa receive the B.Sc. degree in electrical engineering from Pontifical Catholic University, Pocos de Caldas, MG, Brazil, in 2007 and M.Sc in microelectronics from Federal University of Itajuba, Itajuba, MG, Brazil in 2010. Currently, he is researcher in National Institute of Telecommunications in Brazil. His research interests are IoT, digital circuit architecture design, specifically in error correcting codes. 